\title{
Low Grade Appendiceal Mucinous Neoplasm: A Report of Three Cases
}

\author{
Nisha Sharma, Alina Karna, Abhimanyu Jha \\ Department of Pathology, Maharajgunj Medical Campus, Tribhuvan University Teaching Hospital, Kathmandu, \\ Nepal

\section{Corresponding author:}

\section{Nisha Sharma, MBBS, MD} \\ Department of Pathology, Maharajgunj Medical Campus, Tribhuvan University Teaching Hospital, Kathmandu, \\ Nepal \\ Email:drnishasharma@iom.edu.np
}

Submitted : Mar 8, 2020

Accepted : Apr 12, 2020

\begin{abstract}
Low-grade appendiceal mucinous neoplasm (LAMN) is an uncommon malignancy with varied clinical features. The most alarming complication of this neoplasm is seeding of mucin into the adjacent peritoneum leading to pseudomyxoma peritonei (PMP). We report three cases of LAMN. Two cases were seen post appendicectomy in 23-year old and 36-year old females. The third case was seen in 53-year-old male post Whipple procedure and hemicolectomy. LAMNs are often diagnosed incidentally, as clinical presentation is barely specific and resembles acute appendicitis. Hence it is imperative to examine all appendicectomy specimens not to miss cases of appendiceal mucinous neoplasm which can have implications on the further management of the patient.
\end{abstract}

Keywords: Appendectomy, mucinous appendiceal neoplasm, pseudomyxoma peritonei

\section{INTRODUCTION}

L

ow-grade appendiceal mucinous neoplasm (LAMN) is an uncommon malignancy with limited cases reported in the literature so far. It is an incidental diagnosis diagnosed during histopathological examination. It has been reported to account for only $1 \%$ of all Gastro-intestinal (GI) neoplasms. ${ }^{1}$ Likewise, it is found in less than $0.3 \%$ of surgically removed appendices. ${ }^{2}$ Mucinous neoplasms of the appendix are characterized by epithelial proliferation with extracellular mucin and pushing tumor margins. Patients present with an array of clinical features as appendicitis with or without appendiceal perforation, progressive abdominal distension owing to peritoneal dissemination or a palpable mass. ${ }^{3}$

Appendiceal mucinous neoplasms (AMN) are further classified as low-grade or high-grade, based on their cytological features. Histopathological features that characterize LAMNs include bland cytological features, mucin production, hyper mucinous epithelium, limitation to the muscularis propria with loss of underlying lymphoid follicles. LAMNs can grow into the muscularis propria, irritate and cause inflammation of the appendix, and can also lead to appendicular rupture. ${ }^{4}$ High grade appendiceal mucinous neoplasms are characterized by high grade cytological features and pushing margin. ${ }^{3}$ We report here three cases of $L A M N$ along with a review of literature.

\section{CASE 1}

A 23-year-old female presented to the hospital with features of acute appendicitis. Appendicectomy was performed and the specimen was sent for histopathological examination. Gross examination 

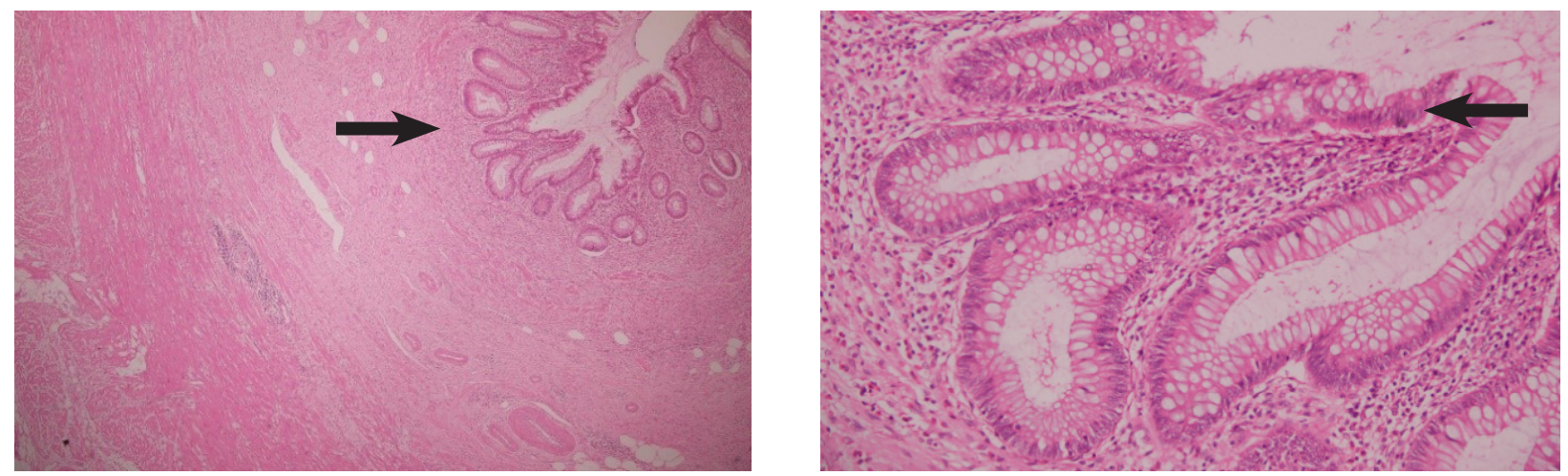

Fig 1a. Hypermucinous epithelium with broad pushing margin and absent lymphoid follicles (H\&E, 40X). Fig 1b. Tumor is lined by pseudostratified mucinous epithelium with minimal atypia and broad pushing margin and extracellular mucin in the lumen (H\&E, 200X).

of the specimen revealed appendix measuring $3 \times 1 \times 0.4 \mathrm{~cm}$. Sectioning of the specimen showed focal mucinous areas. Histopathological examination (H\&E) showed denuded lining with areas of undulating to flattened epithelium lined by mucinous epithelium. Lamina propria showed dense sclerosis with mucin extravasation seen up to muscularis propria. Perivascular and perineural mucinous deposits were also noted in the submucosa and muscularis propria. Hence, the final diagnosis of $\mathrm{pT}$ is (LAMN) was made.

\section{CASE 2}

A 36-year-old female presented with acute appendicular lump. Appendicectomy was performed and the specimen sent for histopathological evaluation. Grossly, appendix measured 5x1 x0.5 $\mathrm{cm}$. Cut section showed thickened wall and distended lumen. Microscopically, mucosa showed mucinous lining with undulation. Tumor was seen obliterating muscularis mucosa at multifocal areas and extending up to submucosa in some of the sections, but muscularis propria was uninvolved

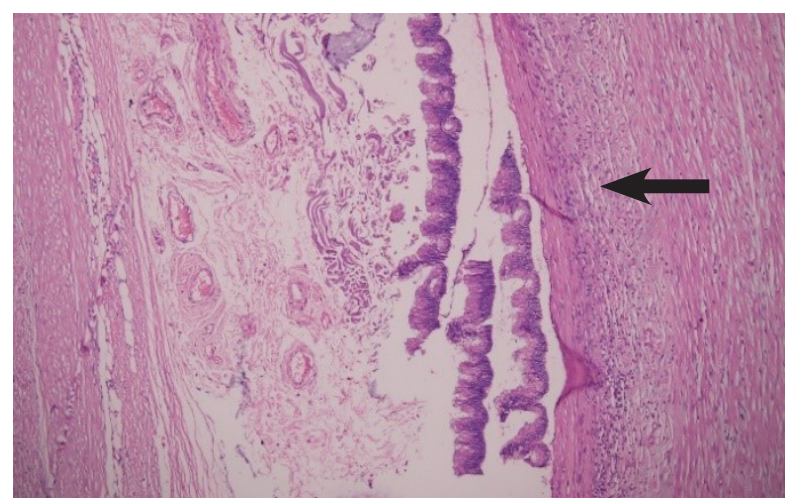

Fig 2. Villous hypermucinous epithelium lined by pseudostratified columnar nuclei replacing the lymphoid tissue. Fibrosis is also noted in the appendiceal wall (H\&E, 100X) by the tumor. Accordingly, a final diagnosis of pTis (LAMN) was made. (Fig. 1a and $1 \mathrm{~b}$ )

\section{CASE 3}

The third case was seen in a 53-year-old male post whipples procedure and hemicolectomy. Computed Tomography (CT) scan showed periampullary mass and a tubular cystic lesion in the appendix. Grossly, appendix was dilated measuring $5 \times 3 \times 0.3$ $\mathrm{cm}$ and filled with mucoid material. Microscopically, mucosa showed mucinous lining with undulation and features of low nuclear atypia. It was limited within the mucosa. A final diagnosis of pTis (LAMN) was made. (Fig. 2)

\section{DISCUSSION}

Appendiceal mucinous neoplasms (AMNs) are very rare tumors accounting for less than $1 \%$ of all cancers with only few cases reported in the literature. ${ }^{5}$ These neoplasms are mostly seen in the sixth decade of life and patients present with varied clinical manifestations ranging from being completely asymptomatic or presenting with acute abdomen and appendicular lump. Such neoplasms are not without notorious complications as intussusception, bowel obstruction, rupture and PMP (seeding of mucin into the peritoneal surface). ${ }^{1,6}$ Mucinous appendiceal tumors behave from being completely benign tumors with very slow rate of progression to high grade adenocarcinomas with speedy recurrence and a dismal prognosis.?

Ultrasonography findings of an elongated or cystic lesion with internal onion-skin appearance, representing mucin, is considered diagnostic of mucinous neoplasms. CT offers even high illustration of appendiceal lesions. ${ }^{2}$ Grossly, appendices may appear totally unremarkable or may be cystically dilated and swollen due to accumulation of mucin. Their potential behavior to spread to the peritoneum and viscera in the form of mucinous deposits 
warrants for proper diagnosis and treatment. 8,9

LAMN consists of cytologically bland flat, filiform, undulating or villous mucinous epithelial proliferation with absence of lymphoid tissue. The wall can show varying degrees of fibrosis, hyalinization and calcification. High grade neoplasms share features of LAMN including subepithelial fibrosis, pushing margin, rupture and peritoneal dissemination. However, the neoplastic epithelium has high grade features with enlarged, hyperchromatic, pleomorphic nuclei and mitotic figures. 3,8

Appendiceal mucinous neoplasms are positive for Cytokeratin 20 (CK20), Mucin 5AC (MUC5AC), Decapentaplegic homolog 4 (DPC4), Mucin 2 (MUC2), CDX2, B-catenin, Carcinoembryonic antigen (CEA) ,Carbohydrate antigen 19-9 (CA 199) and are often negative for Cytokeratin 7 (CK7)., Recent molecular studies have thrown some light into the molecular profiles and mutations associated with the tumor. LAMNs have Kirsten rat sarcoma viral oncogene (KRAS) and Guanine nucleotide binding protein alpha stimulating (GNAS) mutations whereas high grade appendiceal mucinous tumors have decreased KRAS and GNAS alterations and a higher TP53 mutations. ${ }^{10}$ AMNs also follow the same pathogenic pathway as Colorectal cancers (CRCs) involving adenoma-carcinoma sequence and microsatellite instability pathway. ${ }^{5}$

PMP most often occurs as a result of peritoneal spread of a mucinous neoplasm from the appendix but has also been described with mucinous tumors from other sites, including colon, ovary, gallbladder, pancreas etc. ${ }^{8,11} \mathrm{WHO}$ has proposed histological classification of PMP into low grade and high grade. Low cellularity mucin pools $(<10 \%)$, minimal cytological atypia and non-stratified epithelium characterize low grade PMP. On the other hand, features as high cellularity mucin pools, significant cytological atypia with signet ring cells and stromal desmoplasia favor high grade PMP. ${ }^{7}$

Low-grade and high-grade tumors differ from management perspective too. The treatment of lowgrade tumors includes surgical resection in early stage and peritoneal debulking and hyperthermic intraperitoneal chemotherapy (HIPEC) in advanced stage disease. Likewise, high-grade tumors benefit from debulking surgery and HIPEC, with or without preoperative chemotherapy. ${ }^{5,12}$

\section{CONCLUSION}

LAMN is a rare malignancy with worrisome complication of PMP. LAMNs are often diagnosed incidentally, as clinical presentation is barely specific. Those confined to the appendix can often be overlooked and misdiagnosed as appendicitis. Hence it is imperative to keep LAMN in one of the differential diagnoses of acute appendicitis.

\section{CONFLICT OF INTEREST}

None declared.

\section{REFERENCES}

1. Gonzalez HH, Herard K, Mijares MC. A Rare Case of Low-grade Appendiceal Mucinous Neoplasm: A Case Report. Cureus. 2019 Jan 29;11(1):e3980e3980.

2. Tirumani $\mathrm{SH}_{1}$ Fraser-Hill $\mathrm{M}$, Auer $\mathrm{R}$, Shabana W, Walsh $C$, Lee $F$, et al. Mucinous neoplasms of the appendix: a current comprehensive clinicopathologic and imaging review. Cancer Imaging. 2013 Feb 22;13(1):14-25.

3. Misdraji J, Carr N, Pai R. WHO classification of tumours of the digestive system. In: Bosman FT, Carneiro F, Hruban RH, Theise ND, editors. Fifth. Lyon: World Health Organization \& International Agency for Research on Cancer; 2019. p. 144-6.

4. Porter KR, Ramos CE, Neychev V. Low-grade Appendiceal Mucinous Neoplasm in the Context of Acute Appendicitis. Cureus. 2019 Jul 1;11(7):e5159-e5159.

5. Shaib WL, Assi R, Shamseddine A, Alese OB, Staley 3rd C, Memis B, et al. Appendiceal Mucinous Neoplasms: Diagnosis and Management. Oncologist. 2018 Jan;23(1):137.

6. Padmanaban V, Morano WF, Gleeson E, Aggarwal A, Mapow BL, Stein DE, et al. Incidentally discovered low-grade appendiceal mucinous neoplasm: a precursor to pseudomyxoma peritonei. Clin case reports. 2016 Oct;4(12):1112-6.

7. Ramaswamy V. Pathology of Mucinous Appendiceal Tumors and Pseudomyxoma Peritonei. Indian J Surg Oncol. 2016 Jun;7(2):258-67.

8. Misdraji J. Mucinous epithelial neoplasms of the appendix and pseudomyxoma peritonei. Mod Pathol. 2015 Jan;28(1):S67-79.

9. Panarelli NC, Yantiss RK. Mucinous Neoplasms of the Appendix and Peritoneum. Arch Pathol Lab Med. 2011 Oct;135(10):1261-8.

10. Stein A, Strong E, Gamblin TC, Clarke C, Tsai S, Thomas J, et al. Molecular and genetic markers in appendiceal mucinous tumors: a systematic review. Ann Surg Oncol. 2019;1-13.

11. Misdraji J. Appendiceal mucinous neoplasms: controversial issues. Arch Pathol Lab Med. 2010 June;134(6):864-70.

12. Dulskas A, Poskus T, Poskus E, Strupas K. LongTerm Outcomes after Surgery for Appendiceal Mucinous Tumours. Visc Med. 2018 Apr;34(2):1515. 